\title{
Upregulation of gastric adenocarcinoma predictive long intergenic non-coding RNA promotes progression and predicts poor prognosis in perihilar cholangiocarcinoma
}

\author{
XIN-PING WANG ${ }^{1}$, JING SONG ${ }^{2}$, GUI-TING LIU ${ }^{3}$, JIAN-JUN WANG ${ }^{1}$ and HAI-FENG GUO ${ }^{1}$ \\ Departments of ${ }^{1}$ General Surgery, ${ }^{2}$ Urology and ${ }^{3}$ Thoracic Surgery, Hongqi Hospital of Mudanjiang Medical College, \\ Mudanjiang, Heilongjiang 157011, P.R. China
}

Received January 25, 2017; Accepted January 29, 2018

DOI: $10.3892 / \mathrm{ol} .2018 .9137$

\begin{abstract}
Perihilar cholangiocarcinoma (PHCC) is one of the most aggressive and complex types of cancer with a poor survival. Despite advances in PHCC diagnosis and treatment, the biology of this tumor remains poorly understood. Recent studies have suggested long non-coding RNAs (lncRNAs) as crucial determinants of cancer progression. However, the role of lncRNAs in PHCC is rarely reported and the function of gastric adenocarcinoma predictive long intergenic non-coding RNA (GAPLINC) in PHCC has yet to be elucidated. The present study observed a significant upregulation of GAPLINC in PHCC cell lines and clinical specimens $(\mathrm{P}<0.05)$. Furthermore, by comparing clinicopathological characteristics with expression data, high GAPLINC expression was revealed to be associated with the $\mathrm{T}$ stage $(\mathrm{P}=0.013), \mathrm{N}$ stage $(\mathrm{P}<0.001)$ and Tumor-Node-Metastasis stage $(\mathrm{P}<0.001)$ of PHCC. Furthermore, Kaplan-Meier analysis demonstrated that GAPLINC expression was associated with poor overall survival and progression-free survival rates in PHCC. Furthermore, univariate and multivariate COX regression analyses identified high GAPLINC expression as a risk factor of a poor prognosis in PHCC. GAPLINC upregulation promoted the migration and invasion of PHCC cells in Transwell and Matrigel assays, respectively, while GAPLINC deficiency inhibited PHCC cell metastasis. Furthermore, PHCC cells with GAPLINC overexpression exhibited markedly increased proliferation ability in a Cell Counting kit-8 assay. However, GAPLINC interference significantly suppressed cell proliferation. In conclusion, GAPLINC may promote PHCC progression and may serve as a potential prognostic marker and therapeutic target of PHCC.
\end{abstract}

Correspondence to: Professor Jian-Jun Wang, Department of General Surgery, Hongqi Hospital of Mudanjiang Medical College, 5 Tongxiang Road, Mudanjiang, Heilongjiang 157011, P.R. China E-mail: jjwangheilongjiang@163.com

Key words: cholangiocarcinoma, gastric adenocarcinoma predictive long intergenic non-coding RNA, metastasis, prognosis

\section{Introduction}

Cholangiocarcinomas (CCAs), originating from the epithelial cells of the biliary tract, are now divided into intrahepatic CCA, perihilar CCA (PHCC) and distal CCA based on anatomical location, according to a recent study (1). PHCC, accounting for $\sim 50 \%$ of CCAs, is a relatively rare type of carcinoma, but with aggressive characteristics and a poor prognosis. Radical resection is the only effective curative treatment at present. However, the resection rate of PHCC is relatively low as the majority of patients are diagnosed at an advanced stage of its insidious development (2). Additionally, the highly desmoplastic nature, extensive support by a rich tumor microenvironment and profound genetic heterogeneity of PHCC make it resistant to current adjuvant therapeutic strategies (1). Therefore, PHCC remains one of the most difficult challenges for hepatobiliary surgeons (3). In addition, tumor markers, including carbohydrate antigen 19-9 (CA 19-9) and carcinoembryonic antigen (CEA), may be raised in PHCC, but these markers lack the sensitivity required for diagnosis (4). Therefore, further investigations on the underlying molecular mechanisms of PHCC progression, and identifying more effective prognostic biomarkers and therapeutic targets, are urgently required for improving the prognosis of PHCC patients.

Recent improvements in genome-wide surveys and high throughput transcriptome analysis have revealed that the human genome contains only $\sim 20,000$ protein-coding genes, representing $<2 \%$ of the total genome $(5,6)$. While 10 - to 20 -fold more genomic sequence is transcribed to long non-coding RNA (lncRNA) than to protein-coding RNA $(7,8)$. lncRNAs are functionally defined as transcripts $>200$ nucleotides (nt) in length with no protein-coding potential. In recent years, an increasing amount of scientific interest has been focused on investigating the functions and mechanisms of lncRNAs (8-10). Furthermore, it has been suggested that lncRNAs have fulfilled a wide variety of regulatory roles at almost every stage of gene expression, including transcriptional, post-transcriptional and epigenetic levels (9). Furthermore, accumulating evidence has indicated that lncRNAs serve essential roles in cancer development and progression through various regulatory pathways, hierarchies and networks $(11,12)$. Therefore, identification of cancer-associated lncRNAs, and investigation of 
their molecular and biological functions, may shed light on investigating the molecular biology of cancer.

GAPLINC is a recently discovered non-coding RNA and has been revealed to serve essential roles in multiple types of cancer. $\mathrm{Hu}$ et al (13) reported that GAPLINC is involved in gastric cancer progression and may serve as a biomarker. Yang et al (14) demonstrated that GAPLINC may promote invasion in colorectal cancer by targeting snail family zinc finger 2 through binding with PTB-associated splicing factor and non-POU-domain-containing octamerbinding, and may be a potential therapeutic target of colorectal cancer. However, the role of IncRNA GAPLINC in PHCC has yet to be identified.

The present study investigated the expression of GAPLINC in PHCC cell lines and tissues. The association between GAPLINC expression and PHCC clinicopathological features was also investigated. Furthermore, high GAPLINC expression was revealed to be associated with the prognosis of PHCC. GAPLINC may also promote PHCC cell metastasis and proliferation in in vitro assays. These results indicated that GAPLINC may be considered as a potential prognostic marker and therapeutic target for patients with PHCC.

\section{Materials and methods}

Patients and tissue specimens. A total of 96 patients with PHCC who had undergone surgical resection at Hongqi Hospital of Mudanjiang Medical College (Mudanjiang, China) between January 2006 and November 2012 were included in the present

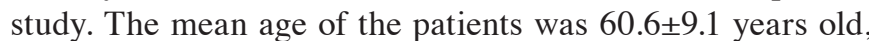
ranging from 41-82. A total of 57 male patients and 39 female patients were involved. The tumor tissues and paired adjacent non-cancerous tissues, isolated from $>1 \mathrm{~cm}$ away from the tumor margin, were collected. It was confirmed by a pathologist at Hongqi Hospital of Mudanjiang Medical College that the adjacent non-cancerous tissues did not contain any clear tumor cells. PHCC tissues and adjacent non-cancerous tissues from each patient were immediately frozen in liquid nitrogen, prior to being stored at $-80^{\circ} \mathrm{C}$ until subsequent RNA extraction. No local or systemic anticancer treatments were administered to these patients prior to surgery. The detailed patient information is presented in Table I. The last follow-up was terminated in June 2016 and the mean follow-up period was 47.8 months (range, 3-100 months). Patients with two or more different malignancies were excluded. Written informed consent was obtained from all patients and the study was approved by the Ethics Committee of Hongqi Hospital of Mudanjiang Medical College.

Cell culture. The human normal biliary epithelial HIBEpiC cell line, and the PHCC QBC939 and FRH0201 cell lines, were purchased from the Cell Bank of Chinese Academy of Sciences (Shanghai, China). Cells were cultured in Dulbecco's modified Eagle's medium (Gibco; Thermo Fisher Scientific, Inc., Waltham, MA, USA) or RPMI-1640 medium (Gibco; Thermo Fisher Scientific, Inc.), supplemented with $10 \%$ fetal bovine serum (FBS; Gibco; Thermo Fisher Scientific, Inc.) and $1 \%$ penicillin/streptomycin (Gibco; Thermo Fisher Scientific, Inc.) at $37^{\circ} \mathrm{C}$ in a humidified incubator with $5 \% \mathrm{CO}_{2}$.
RNA extraction and reverse transcription-quantitative polymerase chain reaction $(R T-q P C R)$. Total RNA was isolated from cell lines or frozen tissue samples using TRIzol reagent (Invitrogen; Thermo Fisher Scientific, Inc.), according to the manufacturer's protocols. DNase I (RNase-free; Takara Biotechnology Co., Ltd., Dalian, China) was used to prevent contamination of DNA and was then inactivated by heat treatment. cDNA was reverse transcribed with the extracted total RNA $(2 \mu \mathrm{g})$ in a total volume of $20 \mu \mathrm{l}$ using the Prime-Script one step RT-PCR kit (Takara Biotechnology Co., Ltd.), the conditions were as follows: Incubation for $5 \mathrm{~min}$ at $25^{\circ} \mathrm{C}$ followed by $60 \mathrm{~min}$ at $42^{\circ} \mathrm{C}$, and then terminatin the reaction by heating at $70^{\circ} \mathrm{C}$ for $5 \mathrm{~min}$. The mRNA level of 1 ncRNA GAPLINC was evaluated by RT-qPCR, which was performed using a SYBR PrimeScript RT-PCR kit (Takara Biotechnology Co., Ltd.) on an ABI7300HT instrument (Applied Biosystems; Thermo Fisher Scientific, Inc.). The PCR thermocycling conditions were as follows: Initial denaturation at $95^{\circ} \mathrm{C}$ for $5 \mathrm{~min}$, followed by 40 cycles of denaturation at $95^{\circ} \mathrm{C}$ for $30 \mathrm{sec}$, annealing at $50^{\circ} \mathrm{C}$ for $30 \mathrm{sec}$ and extension at $72^{\circ} \mathrm{C}$ for $30 \mathrm{sec}$. Each experiment was performed in triplicate. Relative GAPLINC expression level was normalized to GAPDH using the comparative cycle threshold $\left(2^{-\Delta \Delta C q}\right)$ method (15). The primer sequences were as follows: GAPLINC forward, 5'-ACACACAGCAGCCTGGTT TC-3' and reverse, 5'-ATGGCACAATCAGGGCTCTT-3'; and GAPDH forward, 5'-ACCCACTCCTCCACCTTTGAC-3' and reverse, 5'-TGTTGCTGTAGCCAAATTCGTT-3'.

Transfection of cell lines. The GAPLINC sequence was synthesized according to the full-length GAPLINC sequence (based on the GAPLINC sequence on NCBI; https://www .ncbi.nlm.nih.gov/), prior to being sub-cloned into a pcDNA3.1 vector (Genewiz, Inc., Beijing, China). An empty pcDNA3.1 vector was used as the control. Small interfering RNAs (siRNAs) specifically targeting GAPLINC (siGAPLINC-1 and siGAPLINC-2), and a scramble RNA negative control (si-NC) were purchased from Genewiz, Inc. PHCC cells were cultured on 6-well plates to confluency and were transfected using Lipofectamine 2000 (Invitrogen; Thermo Fisher Scientific, Inc.), according to the manufacturer's protocols. At $48 \mathrm{~h}$ post-transfection, the cells were used for in vitro assays.

Transwell and matrigel assays. Migration of QBC939 cells and FRH0201 cells were assessed using a Transwell assay conducted in a Transwell chamber (Corning Incorporated, Corning, NY, USA). After transfection for $24 \mathrm{~h}$, cells $\left(2.5 \times 10^{4}\right)$ were plated into the upper chamber filled with serum-free RPMI-1640 medium (Gibco; Thermo Fisher Scientific, Inc.), while RPMI-1640 medium (Gibco; Thermo Fisher Scientific, Inc.) supplemented with 10\% FBS (Gibco; Thermo Fisher Scientific, Inc.) was added to the lower chamber. Invasion of the cells was examined using the Matrigel method. The Transwell chamber was pre-coated with Matrigel (Corning Incorporated) prior to the cells being plated, followed by incubation of the chamber at $37^{\circ} \mathrm{C}$ for $30 \mathrm{~min}$. After incubation for $24 \mathrm{~h}$ at $37^{\circ} \mathrm{C}$, cells that had migrated to the bottom surface of the membrane were fixed with $4 \%$ paraformaldehyde for $30 \mathrm{~min}$ at room temperature, stained with crystal violet solution for $30 \mathrm{~min}$ at room temperature. Finally, stained cells were visualized under a light microscope (magnification, x100), and 
Table I. Association between GAPLINC expression and clinicopathological parameters of perihilar cholangiocarcinoma.

\begin{tabular}{|c|c|c|c|}
\hline Parameter & $\begin{array}{c}\text { No. } \\
\text { patients }\end{array}$ & $\begin{array}{l}\text { GAPLINC } \\
\text { expression }\end{array}$ & P-value \\
\hline Age, years & & & 0.572 \\
\hline$<65$ & 66 & $4.87 \pm 2.92$ & \\
\hline$\geq 65$ & 30 & $5.26 \pm 3.58$ & \\
\hline Sex & & & 0.836 \\
\hline Male & 57 & $4.94 \pm 3.15$ & \\
\hline Female & 39 & $5.07 \pm 3.14$ & \\
\hline Tumor size, $\mathrm{cm}$ & & & 0.571 \\
\hline$<3$ & 45 & $4.80 \pm 3.07$ & \\
\hline$\geq 3$ & 51 & $5.16 \pm 3.19$ & \\
\hline Differentiation grade & & & 0.716 \\
\hline Well + moderately & 79 & $5.05 \pm 3.11$ & \\
\hline Poorly + undifferentiated & 17 & $4.74 \pm 3.27$ & \\
\hline T stage & & & 0.013 \\
\hline $\mathrm{T} 1+\mathrm{T} 2$ & 78 & $4.62 \pm 3.14$ & \\
\hline $\mathrm{T} 3+\mathrm{T} 4$ & 18 & $6.63 \pm 2.58$ & \\
\hline $\mathrm{N}$ stage & & & $<0.001$ \\
\hline No & 77 & $4.22 \pm 2.84$ & \\
\hline $\mathrm{N} 1+\mathrm{N} 2$ & 19 & $8.11 \pm 2.20$ & \\
\hline M stage & & & 0.564 \\
\hline M0 & 76 & $4.94 \pm 3.11$ & \\
\hline M1 & 3 & $6.53 \pm 4.01$ & \\
\hline TNM stage & & & $<0.001$ \\
\hline $\mathrm{I}+\mathrm{II}$ & 65 & $4.02 \pm 2.87$ & \\
\hline III+IV & 31 & $7.04 \pm 2.65$ & \\
\hline
\end{tabular}

GAPLINC, gastric adenocarcinoma predictive long intergenic non-coding RNA; T, tumor; N, node; M, metastasis.

the number of cells counted in five random fields of view were averaged.

Cell proliferation assay. Cells proliferation was monitored using WST-8 and a Cell Counting kit-8 (CCK-8; Roche Diagnostics $\mathrm{GmbH}$, Mannheim, Germany), according to the manufacturer's protocols. Briefly, 2,000 cells were seeded onto 96-well plates and cell proliferation was monitored every $24 \mathrm{~h}$ for 4 days using a CCK-8 assay. The number of rate of cell proliferation was quantified by reading the absorbance of reduced WST-8 at a wavelength $450 \mathrm{~nm}$.

Statistical analysis. A two-tailed Student's t-test was used to compare two different groups with parametric variables. One-way analysis of variance was used to compare multiple groups. Survival curves were plotted using the Kaplan-Meier method and were compared using the log-rank test. To assess the relative risk for each factor, univariate and multivariate Cox regression analyses were performed. Cut-off scores to discriminate high and low GAPLINC expression samples were selected by evaluating the receiver-operating characteristic (ROC) curves. The point on the curve with the shortest distance to the coordinate $(0,1)$ was selected as the threshold value. All tests were two-sided and $\mathrm{P}<0.05$ was considered to indicate a statistically significant difference. Analysis was performed using the SPSS 19.0 software package (IBM Corp., Armonk, NY, USA).

\section{Results}

Expression of GAPLINC is upregulated in human PHCC. To identify the functional role of GAPLINC in PHCC, RT-qPCR was performed to evaluate its expression in PHCC cell lines. Notably, the PHCC QBC939 and FRH0201 cell lines both exhibited higher expression levels of GAPLINC in comparison with the HIBEpic cell line (Fig. 1A). Furthermore, the expression of GAPLINC in 96 PHCC tissues and paired adjacent non-cancerous tissues was detected. As a result, GAPLINC was more highly expressed in $84.4 \%$ (81/96) PHCC tissues compared with the adjacent non-cancerous tissues. Furthermore, the mean expression level of GAPLINC in PHCC tissues was also much higher than that in tumor adjacent tissues $(1 \pm 0.80$ vs. $1.93 \pm 1.21 ; \mathrm{P}<0.001$; Fig. $1 \mathrm{~B})$. These results suggested that GAPLINC may function as an oncogene in PHCC.

High GAPLINC expression promotes PHCC progression. To extend the present understanding of the role of GAPLINC in PHCC progression, further statistical analyses were performed comparing GAPLINC expression with PHCC clinicopathological characteristics. Patients with advanced $\mathrm{T}$ stage $(\mathrm{P}=0.013)$, $\mathrm{N}$ stage $(\mathrm{P}<0.001)$ and TNM stage $(\mathrm{P}<0.001)$ exhibited significantly higher GAPLINC expression, compared with patients with earlier stages of disease (Fig. 1C-E; Table I). However, no statistically significant associations were observed between GAPLINC expression and other clinicopathological parameters, including age, sex, tumor size, and $\mathrm{M}$ stage $(\mathrm{P}>0.05$; Table I). Taken together, these results indicated that GAPLINC may promote PHCC progression.

Selection of a GAPLINC expression cut-off value for prognosis prediction in PHCC using an ROC curve. To better define the role of GAPLINC expression in the prognosis prediction of patients with PHCC, the ROC curve was employed to identify the optimal cut-off value for high and low expression of GAPLINC. In order to use the ROC curve analysis, the prognosis parameters were dichotomized: Overall survival (OS), death due to PHCC or censored (lost to follow-up, alive or succumbed to mortality from other causes); progression-free survival (PFS), PHCC progressed or censored (lost to follow-up, alive or succumbed to mortality from other causes). As demonstrated in Fig. 2A and B, the area under the ROC curve for OS and PFS was 0.713 (95\% CI, 0.597-0.829), which indicated that GAPLINC may serve as a valuable prognostic marker for patients with PHCC $(\mathrm{P}<0.001)$. The cut-off value of the OS and PFS for high and low GAPLINC expression was 3.10. Based on the obtained cut-off value, the patients were classified into two groups, the high GAPLINC expression group $(n=57)$ and the low GAPLINC expression group $(n=39)$.

GAPLINC overexpression predicts a poor prognosis in $P H C C$. To further verify the prognostic potential of GAPLINC expression in PHCC, Kaplan-Meier analysis was 
A

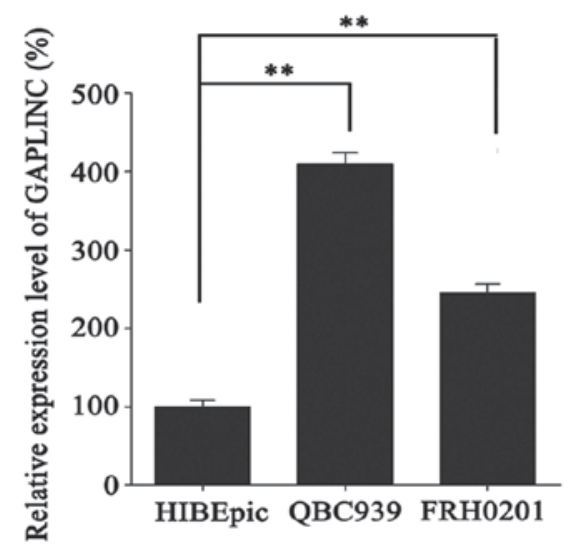

B

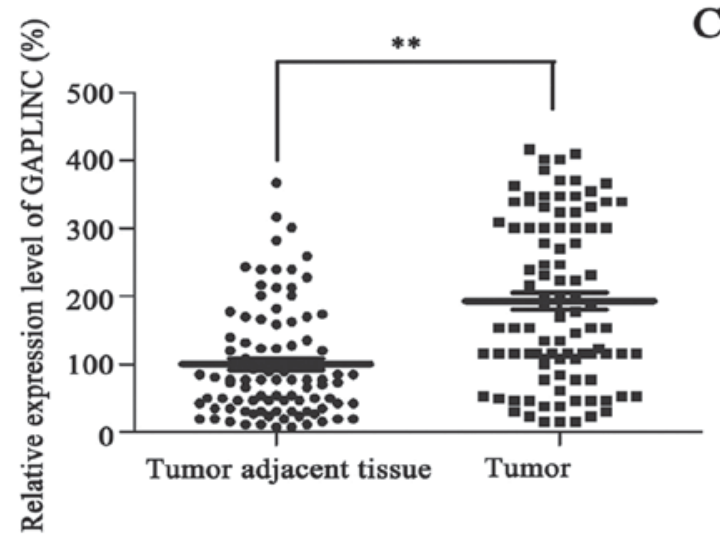

D

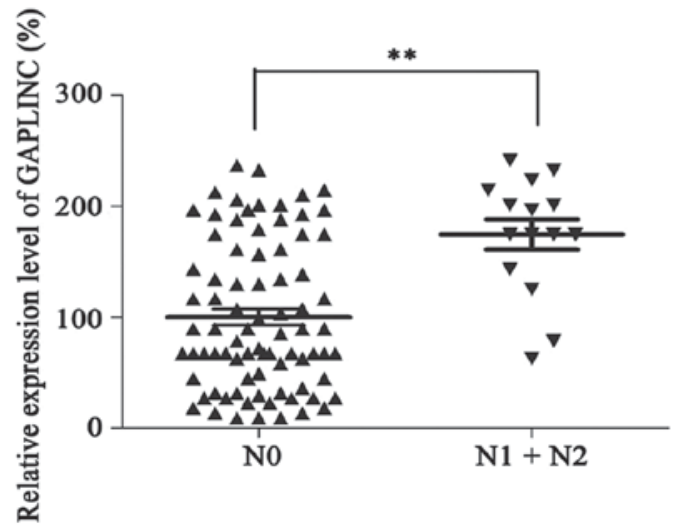

C

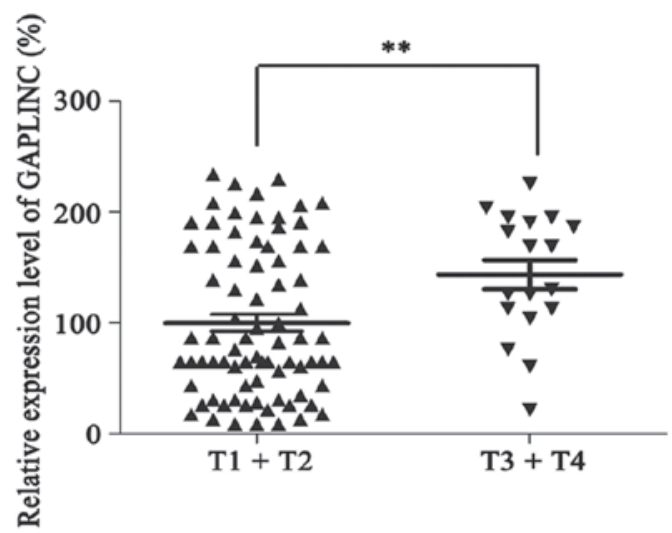

E

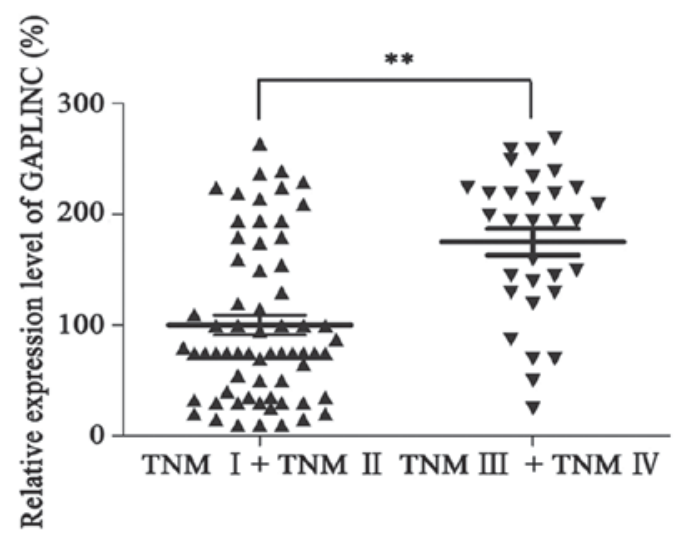

Figure 1. GAPLINC is overexpressed in PHCC. (A) RT-qPCR was performed to detect the expression of GAPLINC in PHCC cell lines and normal biliary epithelial HIBEpic cells. (B) GAPLINC expression was analyzed in 96 PHCC tissues and paired adjacent non-cancerous tissues by RT-qPCR assay. (C) The expression levels of GAPLINC were compared using RT-qPCR in patients with T1 + T2 and T3 + T4 disease. (D) The expression levels of GAPLINC were compared using RT-qPCR in patients with N0 and N1 + N2 disease. (E) The expression levels of GAPLINC were compared using RT-qPCR for patients with TNM I + TNM II and TNM III + TNM IV disease. (A) ${ }^{* *} \mathrm{P}<0.01$, using one-way analysis of variance. (B-E) ${ }^{* * *} \mathrm{P}<0.01$, using Student's t-test. Data are presented as the mean \pm standard deviation. GAPLINC, gastric adenocarcinoma predictive long intergenic non-coding RNA; PHCC, perihilar cholangiocarcinoma; RT-qPCR, reverse transcription-quantitative polymerase chain reaction; T, tumor; N, node; TNM, Tumor-Node-Metastasis.

performed, which demonstrated that high GAPLINC expression was associated with a lower OS rate $(\mathrm{P}<0.001$; Fig. $2 \mathrm{C})$. Furthermore, high GAPLINC expression was associated with a poorer PFS $(\mathrm{P}<0.001$; Fig. 2D). Univariate analysis identified the following 4 risk factors for OS: Differentiation $(\mathrm{HR}=0.306 ; 95 \% \mathrm{CI}, 0.122-0.767 ; \mathrm{P}=0.012), \mathrm{N}$ stage $(\mathrm{HR}=2.779 ; 95 \% \mathrm{CI}, 1.554-4.970 ; \mathrm{P}=0.001)$, TNM stage $(\mathrm{HR}=2.503 ; 95 \% \mathrm{CI}, 1.475-4.250 ; \mathrm{P}=0.001)$ and GAPLINC expression level $(\mathrm{HR}=4.655$; 95\% CI, 2.514-8.621; $\mathrm{P}<0.001)$. Furthermore, the same 4 risk factors were identified for PFS:
Differentiation $(\mathrm{HR}=0.319 ; 95 \% \mathrm{CI}, 0.137-0.743 ; \mathrm{P}=0.008), \mathrm{N}$ stage $(\mathrm{HR}=2.667 ; 95 \% \mathrm{CI}, 1.496-4.754 ; \mathrm{P}=0.001)$, TNM stage $(\mathrm{HR}=2.302 ; 95 \% \mathrm{CI}, 1.372-3.862 ; \mathrm{P}=0.002)$ and GAPLINC expression level ( $\mathrm{HR}=3.839 ;$ 95\% CI, 2.191-6.727; $\mathrm{P}<0.001)$. Other clinicopathological features, including sex and age, were not statistically significant prognosis factors (Table II). Furthermore, by performing multivariate Cox regression analysis on these 4 factors, the following 3 prognostic factors were revealed to be independent risk factors for OS: Differentiation $(\mathrm{HR}=0.154 ; 95 \% \mathrm{CI}, 0.054-0.437 ; \mathrm{P}<0.001)$, 

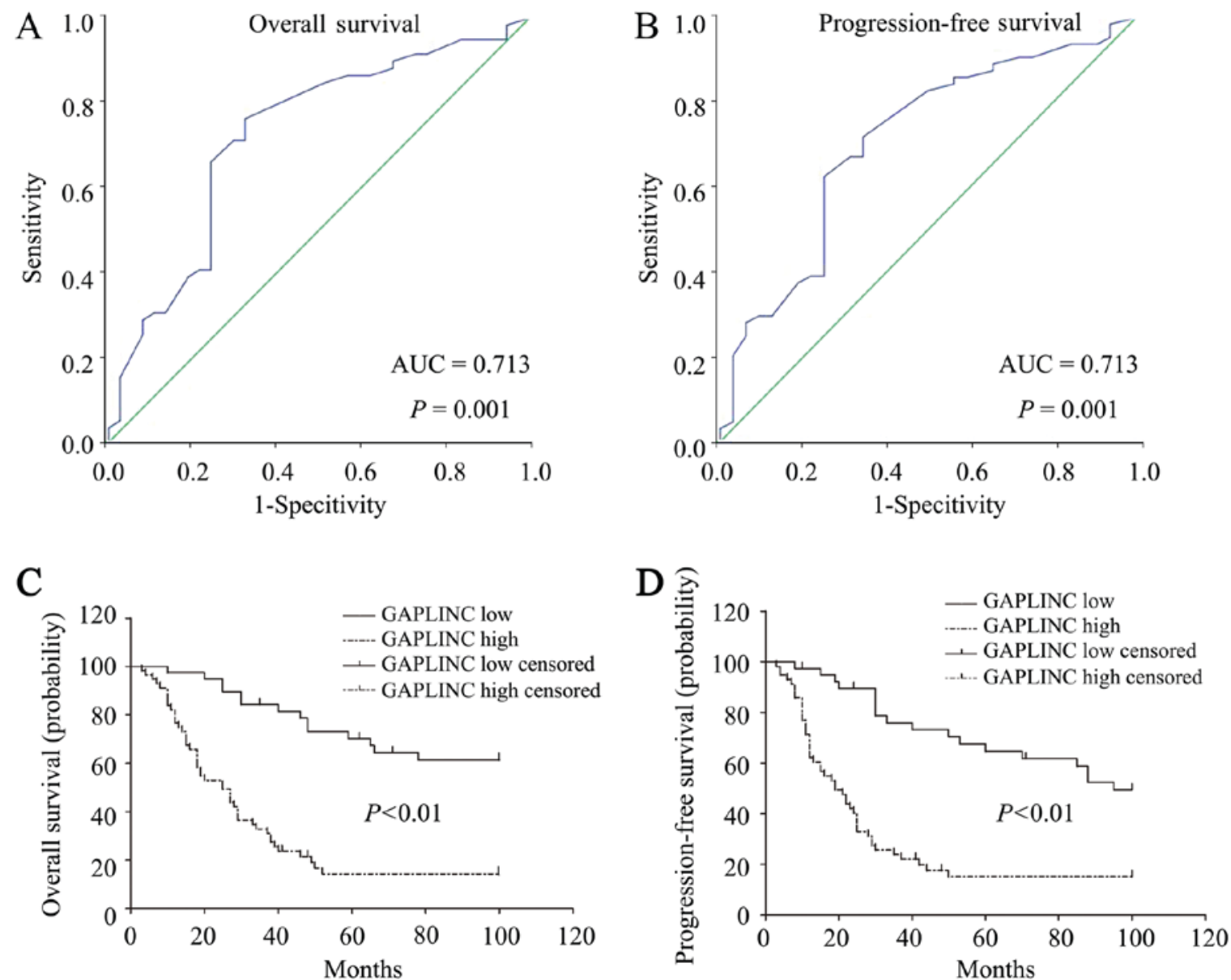

Figure 2. GAPLINC is associated with a poor prognosis in patients with PHCC. The cut-off points of GAPLINC expression for the (A) OS and (B) PFS were analyzed using a receiver operating characteristic curve. Kaplan-Meier analysis and the log-rank test were adopted to investigate the (C) OS and (D) PFS differences between the low GAPLINC expression group and the high GAPLINC expression group. GAPLINC, gastric adenocarcinoma predictive long intergenic non-coding RNA; PHCC, perihilar cholangiocarcinoma; OS, overall survival; PFS, progression-free survival; AUC, area under the curve.

TNM stage $(\mathrm{HR}=3.464 ; 95 \% \mathrm{CI}, 0.173-1.259 ; \mathrm{P}=0.011)$ and GAPLINC expression level (HR=4.470; 95\% CI, 2.269-8.807; $\mathrm{P}<0.001)$, and the same 3 factors were identified for PFS: Differentiation $(\mathrm{HR}=0.167 ; 95 \% \mathrm{CI}, 0.064-0.435 ; \mathrm{P}<0.001)$, TNM stage $(\mathrm{HR}=3.164 ; 95 \% \mathrm{CI}, 1.240-8.072 ; \mathrm{P}=0.016)$ and GAPLINC expression $(\mathrm{HR}=3.804 ; 95 \% \mathrm{CI}, 2.044-7.078$; $\mathrm{P}<0.001$; Table II). Taken together, these results suggested that GAPLINC may be considered as a predictive factor for poor survival and early tumor recurrence in patients with PHCC.

GAPLINC may facilitate the metastasis and proliferation of PHCC cells. To further verify the function of GAPLINC in PHCC, GAPLINC was knocked down and overexpressed. siNC, siGAPLINC-1 and siGAPLINC-2 were used in QBC939 cells, while vector and GAPLINC were used in FRH0201 cells, as QBC939 cells exhibit a comparatively higher GAPLINC expression than FRH0201 (Fig. 1A). Therefore, GAPLINC silencing in QBC939 cells and ectopic expression of GAPLINC in FRH0201 cells would be more reliable for examining the functional role of GAPLINC in PHCC cells. The expression of GAPLINC in QBC939 cells and FRH0201 cells following transfection was determined using RT-qPCR (Fig. 3A and B). The results of the Transwell (Fig. 3C and D) and Matrigel assays demonstrated that GAPLINC interference in QBC939 cells repressed the cell migration and invasion abilities markedly. Accordingly, GAPLINC ectopic expression in FRH0201 cells markedly increased metastasis (Fig. 3E and F). QBC939 cells with GAPLINC deficiency exhibited much lower proliferation ability in a CCK-8 assay (Fig. 4A), while GAPLINC upregulation in FRH0201 cells markedly promoted proliferation (Fig. 4B). These results indicated that GALINC may promote the metastasis and proliferation of PHCC cells.

\section{Discussion}

Previous studies have focused on the diagnosis and management of PHCC, and numerous advances have been made in recent decades $(3,16)$. However, standard adjuvant therapeutic strategies remain unavailable for patients with PHCC. Furthermore, due to the relatively low incidence rate, alterations in classification, diagnosis and treatment strategies and the highly aggressive nature of the disease, the vast majority of published studies on PHCC are statically underpowered, nonrandomized, restricted to short-term follow-up or demonstrate a poor response rate (16). GAPLINC was selected for the present study as it has been demonstrated to promote the progression of gastric and colorectal cancer, and 
Table II. Multivariate analysis of clinicopathological features for overall survival and progression-free survival in patients with perihilar cholangiocarcinoma.

\begin{tabular}{|c|c|c|c|c|c|c|}
\hline \multirow[b]{2}{*}{ Parameter } & \multicolumn{3}{|c|}{ Overall survival } & \multicolumn{3}{|c|}{ Progression-free survival } \\
\hline & HR & $95 \%$ CI & P-value & HR & $95 \% \mathrm{CI}$ & P-value \\
\hline \multicolumn{7}{|l|}{ Univariate analysis } \\
\hline \multicolumn{7}{|l|}{ Age } \\
\hline$\geq 65$ years vs. $<65$ years & 1.321 & $0.779-2.240$ & 0.302 & 1.194 & $0.712-2.002$ & 0.501 \\
\hline \multicolumn{7}{|l|}{ Sex } \\
\hline Male vs. Female & 1.033 & $0.614-1.738$ & 0.901 & 0.963 & $0.583-1.593$ & 0.884 \\
\hline \multicolumn{7}{|l|}{ Differentiation } \\
\hline Well + Moderately vs. Poorly + Undifferentiated & 0.306 & $0.122-0.767$ & 0.012 & 0.319 & $0.137-0.743$ & 0.008 \\
\hline $\begin{array}{l}\text { Tumor size } \\
\geq 3 \mathrm{~cm} \text { vs. }<3 \mathrm{~cm}\end{array}$ & 1.125 & $0.673-1.125$ & 0.653 & 1.066 & $0.651-1.745$ & 0.801 \\
\hline \multicolumn{7}{|l|}{ T stage } \\
\hline $\mathrm{T} 3+\mathrm{T} 4$ vs. $\mathrm{T} 1+\mathrm{T} 2$ & 1.712 & $0.922-3.179$ & 0.089 & 1.516 & $0.822-2.795$ & 0.183 \\
\hline \multicolumn{7}{|l|}{$\mathrm{N}$ stage } \\
\hline $\mathrm{N} 1+\mathrm{N} 2$ vs. N0 & 2.779 & $1.554-4.970$ & 0.001 & 2.667 & $1.496-4.754$ & 0.001 \\
\hline \multicolumn{7}{|l|}{ M stage } \\
\hline M1 vs. M0 & 3.149 & $0.402-4.691$ & 0.275 & 2.043 & $0.270-5.475$ & 0.489 \\
\hline \multicolumn{7}{|l|}{ TNM stage } \\
\hline \multicolumn{7}{|l|}{ GAPLINC expression } \\
\hline High vs. Low & 4.655 & $2.514-8.621$ & $<0.001$ & 3.839 & $2.191-6.727$ & $<0.001$ \\
\hline \multicolumn{7}{|l|}{ Multivariate analysis } \\
\hline \multicolumn{7}{|l|}{ Differentiation } \\
\hline Well + Moderately vs. Poorly + Undifferentiated & 0.154 & $0.054-0.437$ & $<0.001$ & 0.167 & $0.064-0.435$ & $<0.001$ \\
\hline \multicolumn{7}{|l|}{$\mathrm{N}$ stage } \\
\hline $\mathrm{N} 1+\mathrm{N} 2$ vs. N0 & 0.466 & $0.173-1.259$ & 0.132 & 0.530 & $0.200-1.406$ & 0.202 \\
\hline \multicolumn{7}{|l|}{ TNM stage } \\
\hline \multicolumn{7}{|l|}{ GAPLINC expression } \\
\hline High vs. Low & 4.470 & $2.269-8.807$ & $<0.001$ & 3.804 & 2.044-7.078 & $<0.001$ \\
\hline
\end{tabular}

HR, hazard ratio; CI, confidence interval; T, tumor; N, node; M, metastasis; GAPLINC, gastric adenocarcinoma predictive long intergenic non-coding RNA.

to be associated with cancer prognosis $(13,14)$. PHCC also originates from the epithelium cancer, as with gastric and colorectal cancer. However, the functional role of GAPLINC in PHCC has yet to be elucidated. The present study collected 96 PHCC specimens and the prognosis of these patients was determined. Clinicopathological features of these patients were also reviewed and statistical analysis was performed to verify the functional role of GAPLINC in the progression of PHCC. IncRNA GAPLINC overexpression was revealed to promote tumor progression by being associated with advanced T, N and TNM stages of PHCC. Furthermore, ROC curves were constructed and the optimal cut-off value of GAPLINC expression in the prognostic prediction of PHCC patients was defined. Based on the cut-off value, the patients were divided into a high GAPLINC expression group $(57 / 96,59.4 \%)$ and a low GAPLINC expression group (39/96, 40.6\%). The percentage of patients with PHCC exhibiting a high expression of GAPLINC was relatively higher compared than the number reported in patients with colorectal cancer $(85 / 180,47.2 \%)$ and gastric cancer $(45 / 90,50.0 \%)(13,14)$. The log-rank test revealed that high GAPLINC expression predicted lower OS and PFS rates. Furthermore, univariate and multivariate Cox regression analyses revealed that high GAPLINC expression was an independent risk factor for lower OS and PFS rates in patients with PHCC, indicating that GAPLINC may be considered as a survival and recurrence biomarker in patients with PHCC.

The number of lncRNAs identified in recent years has markedly increased, so too has the number of lncRNAs involved in cancer biology. Certain lncRNAs, including GAS5 $(17,18)$, PTENP1 (19), HOTAIR (20,21), MALAT1 (22-24) and 
A

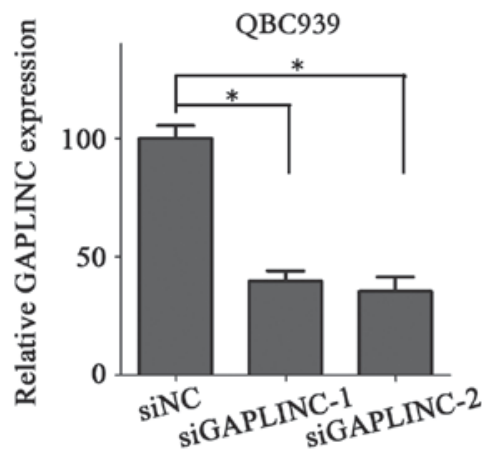

C sinc

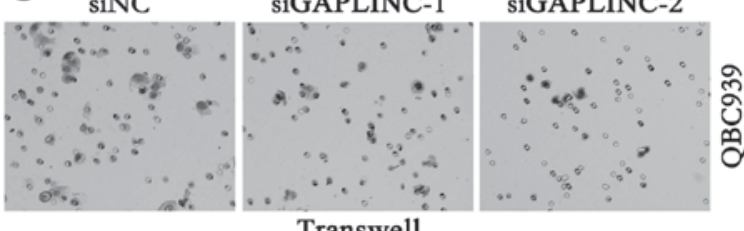

Transwell

$\mathrm{D}$

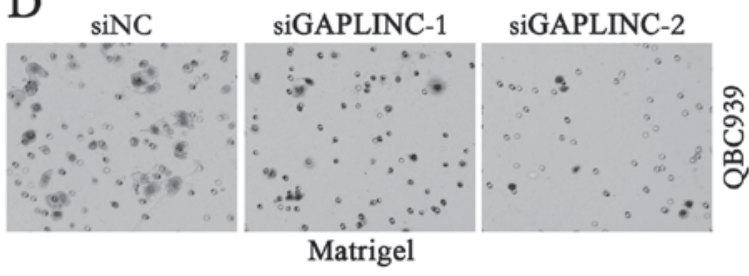

B
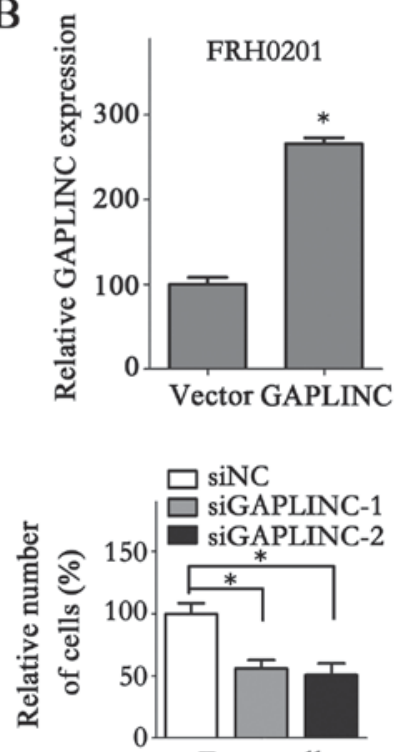

Transwell

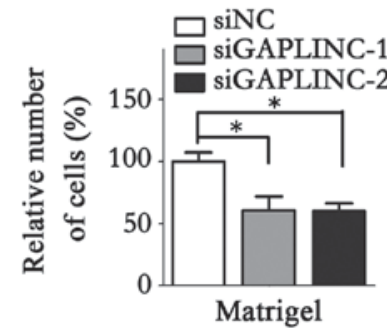

$\mathrm{E}$
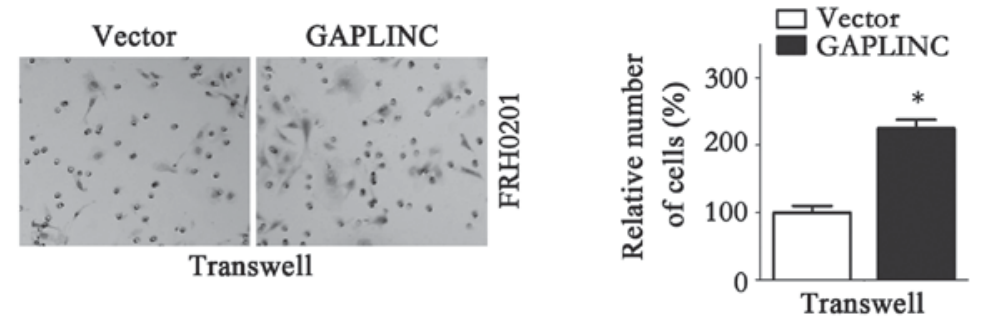

F
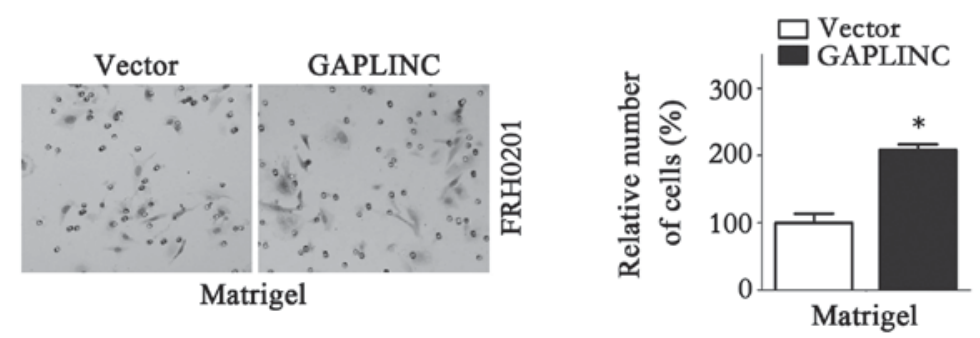

Figure 3. GAPLINC promoted the migration and invasion of PHCC cells. (A) The expression of GAPLINC in QBC393 cells was examined by RT-qPCR following GAPLINC interference. (B) RT-qPCR was used to evaluate the expression level of GAPLINC in FRH0201 cells following GAPLINC overexpression. (C) The migration ability of QBC939 cells with GAPLINC deficiency was assessed using a Transwell assay. (D) The invasive ability of QBC939 cells with GAPLINC silencing was examined using a Matrigel assay. (E) The migration ability of FRH0201 cells with GAPLINC overexpression was assessed using a Transwell assay. (F) The invasion ability of FRH0201 cells with GAPLINC upregulation was examined using a Matrigel assay. (A, C and D) *P $<0.01$, using one-way analysis of variance. (B, E and F) * $\mathrm{P}<0.05$, using Student's t-test. Data are presented as the mean \pm standard deviation. GAPLINC, gastric adenocarcinoma predictive long intergenic non-coding RNA; PHCC, perihilar cholangiocarcinoma; RT-qPCR, reverse transcription-quantitative polymerase chain reaction.

PCAT1 $(25,26)$, have been revealed to be associated with various types of cancer. However, studies on the role of lncRNAs in PHCC are relatively rare (27-29). Wang et al (27) revealed that IncRNA H19 and HULC, upregulated by oxidative stress, may regulate CCA cell migration and invasion by targeting interleukin 6 and C-X-C chemokine receptor type 4 (CXCR4) via competing endogenous RNA patterns of sponging let-7a/let-7b and microRNA (miR)-372/miR-373, respectively. Ma et al (28) demonstrated that the expression levels of carbamoyl phosphate synthase 1 (CPS1) and its long non-coding RNA, CPS1-intronic 

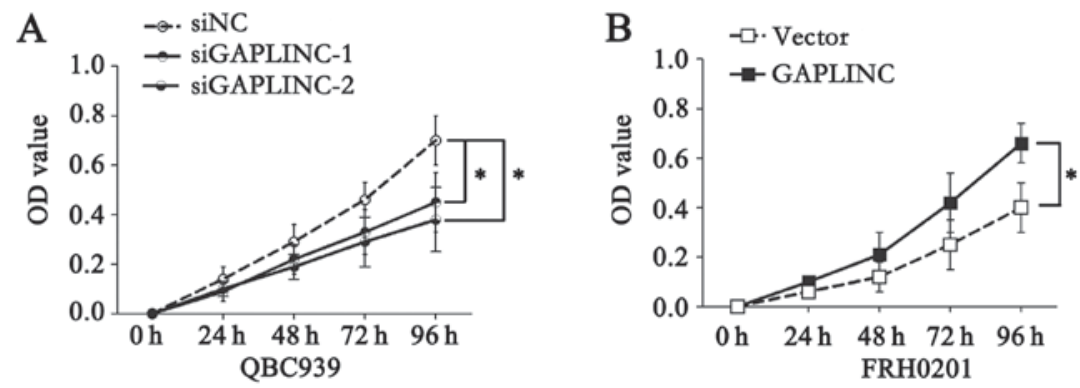

Figure 4. GAPLINC upregulated the proliferation ability of PHCC cells. (A) A CCK-8 assay was performed to evaluate the proliferation ability of QBC939 cells following GAPLINC knock-down. (B) The proliferation ability of FRH0201 cells with GAPLINC overexpression was assessed by a CCK-8 assay. (A) ${ }^{*} \mathrm{P}<0.01$, using the one-way analysis of variance. (B) " $\mathrm{P}<0.05$, using Student's $t$-test. Data are presented as the mean \pm standard deviation. GAPLINC, gastric adenocarcinoma predictive long intergenic non-coding RNA; PHCC, perihilar cholangiocarcinoma; CCK-8, Cell Counting kit-8.

transcript 1, were increased in intrahepatic CCA tissues and cell lines. Additionally, overexpression of CPS1 promoted tumor proliferation and was associated with poor liver function and a poorer prognosis in patients with intrahepatic CCA. Tan et al (29) indicated that lncRNA metastasis-associated lung adenocarcinoma transcript 1 may interact with miR-204 to modulate PHCC proliferation, migration and invasion by targeting CXCR4. These studies have provided novel insight into the roles of lncRNAs in the diagnosis and treatment of PHCC. Therefore, the clinical significance of other lncRNAs, including H19, HULC and CPS1-IT1 has been verified in previous studies. The present study aimed to define the role of GAPLINC in PHCC and therefore, the expression status of other IncRNAs was not determined in the present study. The results of the present study demonstrated that GAPLINC is upregulated in PHCC cell lines and patients tissues, and that there is an association between GAPLINC expression and PHCC clinicopathological characteristics, OS and PFS. Furthermore, high GAPLINC expression was demonstrated to be an independent risk factor for PHCC, indicating that GAPLINC may also be an effective therapeutic target for patients with PHCC. In vitro assays further revealed that GAPLINC promoted the metastasis and proliferation of PHCC cells.

Several different types of molecules have been suggested to be potential diagnostic markers or therapeutic targets for patients with cancer. However, few of them have been commonly used in clinical practice due to relatively low specificity and/or sensitivity. In the present study, the clinical samples were staged based on the TNM staging criteria, a commonly used method for evaluating tumor development in clinical practice at present. No previous studies have verified that the expression levels of existing tumor biomarkers are significantly associated with the PHCC stage. CEA and CA 19-9 are widely used in the diagnosis of PHCC, but recent studies have reported that they lack sufficient specificity and sensitivity (4). Therefore, the clinical samples were not further stained using these tumor markers in the present study. However, the association between GAPLINC expression and certain commonly used tumor markers require further investigation in future studies. IncRNAs may be detected in human plasma, which is a non-invasive way to achieve early diagnosis compared with diagnosing using protein-coding genes, which usually requires invasive manipulation to obtain clinical samples. Certain lncRNAs, including H19, have been revealed to have strong potential as blood biomarkers for the diagnosis of cancer (30). Furthermore, lncRNAs exhibit cell-specific expression patterns to a greater degree than mRNA and usually possess an evolutionarily conserved function and secondary structure, making lncRNAs more appropriate for use as diagnostic and prognostic markers.

In conclusion, the present study confirmed that the expression of GAPLINC was upregulated in PHCC tissues compared with expression in paired adjacent non-cancerous tissues. Furthermore, GAPLINC overexpression may promote the development of PHCC. In addition, high GAPLINC expression predicts a poor OS rate and an early recurrence in patients with PHCC, and serves as an independent risk factor for PHCC prognosis. Further analysis demonstrated that GAPLINC may promote the metastasis and proliferation of PHCC cells. All these results indicated that GAPLINC may be a prognostic maker and therapeutic target in PHCC.

\section{Acknowledgements}

Not applicable.

\section{Funding}

Not applicable.

\section{Availability of data and materials}

The datasets used and/or analyzed during the current study are available from the corresponding author on reasonable request.

\section{Authors' contributions}

XW performed the in vitro assays and the RT-qPCR assays. JS and GL collected the PHCC tissues and performed the statistical analysis. HG was involved in designing the study, collection of tissues, analysis of the data, drafting the manuscript, revising it critically for important intellectual content, and gave final approval of the version to be published and JW designed the present study.

\section{Ethics approval and consent to participate}

Written informed consent was obtained from all patients and the study was approved by the Ethics Committee of Hongqi Hospital of Mudanjiang Medical College (Mudanjiang, China). 


\section{Patient consent for publication}

Written informed consent was obtained from all patients.

\section{Competing interests}

The authors declare that they have no competing interests.

\section{References}

1. Razumilava N and Gores GJ: Cholangiocarcinoma. Lancet 383: 2168-2179, 2014.

2. Khan SA, Davidson BR, Goldin RD, Heaton N, Karani J, Pereira SP, Rosenberg WM, Tait P, Taylor-Robinson SD, Thillainayagam AV, et al: Guidelines for the diagnosis and treatment of cholangiocarcinoma: An update. Gut 61: 1657-1669, 2012.

3. Zhimin G, Noor H, Jian-Bo Z, Lin W and Jha RK: Advances in diagnosis and treatment of hilar cholangiocarcinoma-a review. Med Sci Monit 19: 648-656, 2013.

4. Patel T: Cholangiocarcinoma-controversies and challenges. Nat Rev Gastroenterol Hepatol 8: 189-200, 2011.

5. Guttman M, Amit I, Garber M, French C, Lin MF, Feldser D, Huarte M,Zuk O, Carey BW, Cassady JP, et al: Chromatin signature reveals over a thousand highly conserved large non-coding RNAs in mammals. Nature 458: 223-227, 2009.

6. ENCODEProject Consortium, Birney E, Stamatoyannopoulos JA, Dutta A, Guigó R, Gingeras TR, Margulies EH, Weng Z, Snyder M, Dermitzakis ET, et al: Identification and analysis of functional elements in 1\% of the human genome by the ENCODE pilot project. Nature 447: 799-816, 2007.

7. Nagano $\mathrm{T}$ and Fraser P: No-nonsense functions for long noncoding RNAs. Cell 145: 178-181, 2011.

8. Ponting CP, Oliver PL and Reik W: Evolution and functions of long noncoding RNAs. Cell 136: 629-641, 2009.

9. Li X, Wu Z, Fu X and Han W: IncRNAs: Insights into their function and mechanics in underlying disorders. Mutat Res Rev Mutat Res 762: 1-21, 2014.

10. Cheetham SW, Gruhl F, Mattick JS and Dinger ME: Long noncoding RNAs and the genetics of cancer. Br J Cancer 108: 2419-2425, 2013.

11. Evans JR, Feng FY and Chinnaiyan AM: The bright side of dark matter: lncRNAs in cancer. J Clin Invest 126: 2775-2782, 2016.

12. Serviss JT, Johnsson P and Grander D: An emerging role for long non-coding RNAs in cancer metastasis. Front Genet 5: 234, 2014.

13. Hu Y, Wang J, Qian J, Kong X, Tang J, Wang Y, Chen H, Hong J, Zou W, Chen Y, et al: Long noncoding RNA GAPLINC regulates CD44-dependent cell invasiveness and associates with poor prognosis of gastric cancer. Cancer Res 74: 6890-6902, 2014.

14. Yang $\mathrm{P}, \mathrm{Chen} \mathrm{T}, \mathrm{Xu} \mathrm{Z}$, Zhu H, Wang J and He Z: Long noncoding RNA GAPLINC promotes invasion in colorectal cancer by targeting SNAI2 through binding with PSF and NONO. Oncotarget 7: 42183-42194, 2016.

15. Livak KJ and Schmittgen TD: Analysis of relative gene expression data using real-time quantitative PCR and the 2(-Delta Delta C(T)) method. Methods 25: 402-408, 2001
16. Howell M and Valle JW: The role of adjuvant chemotherapy and radiotherapy for cholangiocarcinoma. Best Pract Res Clin Gastroenterol 29: 333-343, 2015.

17. Mourtada-Maarabouni M, Pickard MR, Hedge VL, Farzaneh F and Williams GT: GAS5, a non-protein-coding RNA, controls apoptosis and is downregulated in breast cancer. Oncogene 28: 195-208, 2009.

18. Xing Z, Lin A, Li C, Liang K, Wang S, Liu Y, Park PK, Qin L, Wei Y, Hawke DH, et al: lncRNA directs cooperative epigenetic regulation downstream of chemokine signals. Cell 159: 1110-1125, 2014.

19. Poliseno L, Salmena L, Zhang J, Carver B, Haveman WJ and Pandolfi PP: A coding-independent function of gene and pseudogene mRNAs regulates tumour biology. Nature 465: 1033-1038, 2010.

20. Gupta RA, Shah N, Wang KC, Kim J, Horlings HM, Wong DJ, Tsai MC, Hung T, Argani P, Rinn JL, et al: Long non-coding RNA HOTAIR reprograms chromatin state to promote cancer metastasis. Nature 464: 1071-1076, 2010.

21. Li L, Liu B, Wapinski OL, Tsai MC, Qu K, Zhang J, Carlson JC, Lin M, Fang F, Gupta RA, et al: Targeted disruption of Hotair leads to homeotic transformation and gene derepression. Cell Rep 5: 3-12, 2013.

22. Ji P, Diederichs S, Wang W, Böing S, Metzger R, Schneider PM, Tidow N, Brandt B, Buerger H, Bulk E, et al: MALAT-1, a novel noncoding RNA, and thymosin beta4 predict metastasis and survival in early-stage non-small cell lung cancer. Oncogene 22: 8031-8041, 2003.

23. Gutschner T, Hämmerle $M$ and Diederichs S: MALAT1-a paradigm for long noncoding RNA function in cancer. J Mol Med (Berl) 91: 791-801, 2013.

24. Tripathi V, Ellis JD, Shen Z, Song DY, Pan Q, Watt AT, Freier SM, Bennett CF, Sharma A, Bubulya PA, et al: The nuclear-retained noncoding RNA MALAT1 regulates alternative splicing by modulating SR splicing factor phosphorylation. Mol Cell 39: 925-938, 2010.

25. Prensner JR, Iyer MK, Balbin OA, Dhanasekaran SM, Cao Q, Brenner JC, Laxman B, Asangani IA, Grasso CS, Kominsky HD, et al: Transcriptome sequencing across a prostate cancer cohort identifies PCAT-1, an unannotated lincRNA implicated in disease progression. Nat Biotechnol 29: 742-749, 2011.

26. Prensner JR, Chen W, Han S, Iyer MK, Cao Q, Kothari V, Evans JR, Knudsen KE, Paulsen MT, Ljungman M, et al: The long non-coding RNA PCAT-1 promotes prostate cancer cell proliferation through cMyc. Neoplasia 16: 900-908, 2014.

27. Wang WT, Ye H, Wei PP, Han BW, He B, Chen ZH and Chen YQ: LncRNAs H19 and HULC, activated by oxidative stress, promote cell migration and invasion in cholangiocarcinoma through a ceRNA manner. J Hematol Oncol 9: 117, 2016.

28. Ma SL, Li AJ, Hu ZY, Shang FS and Wu MC: Co-expression of the carbamoyl-phosphate synthase 1 gene and its long non-coding RNA correlates with poor prognosis of patients with intrahepatic cholangiocarcinoma. Mol Med Rep 12: 7915-7926, 2015.

29. Tan X, Huang Z and Li X: Long non-coding RNA MALAT1 interacted with miR-204 to modulates human Hilar cholangiocarcinoma proliferation, migration and invasion by targeting CXCR4. J Cell Biochem 118: 3643-3653, 2017.

30. Gan L, Xu M, Zhang Y, Zhang X and Guo W: Focusing on long noncoding RNA dysregulation in gastric cancer. Tumour Biol 36: $129-141,2015$. 